\title{
MANHOLE COVER DETECTION USING VEHICLE-BASED MULTI-SENSOR DATA
}

\author{
Ji Shunping ${ }^{\text {a }}$, Shi Yun ${ }^{\text {b, }}$, Shi Zhongchao ${ }^{c}$ \\ ${ }^{\text {a }}$ School of Remote Sensing and Information Engineering, Wuhan University, Wuhan 430049, China \\ jishunping2000@163.com \\ ${ }^{\mathrm{b}}$ CSIS, the University of Tokyo, Tokyo, Japan \\ shiyun@iis.u-tokyo.ac.jp \\ ${ }^{\mathrm{c}}$ Dept. of Environmental and Information Studies, Tokyo City University, Yokohama, Japan \\ shizc@tcu.ac.jp
}

III/4: Complex Scene Analysis and 3D Reconstruction

KEY WORDS: cover detection; matching; edge detection; manhole cover; multi-sensor

\begin{abstract}
:
A new method combined with multi-view matching and feature extraction technique is developed to detect manhole covers on the streets using close-range images combined with GPS/IMU and LIDAR data. The covers are an important target on the road traffic as same as transport signs, traffic lights and zebra crossing but with more unified shapes. However, the different shoot angle and distance, ground material, complex street scene especially its shadow, and cars in the road have a great impact on the cover detection rate. The paper introduces a new method in edge detection and feature extraction in order to overcome these difficulties and greatly improve the detection rate. The LIDAR data are used to do scene segmentation and the street scene and cars are excluded from the roads. And edge detection method base on canny which sensitive to arcs and ellipses is applied on the segmented road scene and the interesting areas contain arcs are extracted and fitted to ellipse. The ellipses are then resampled for invariance to shooting angle and distance and then are matched to adjacent images for further checking if covers and. More than 1000 images with different scenes are used in our tests and the detection rate is analyzed. The results verified our method have its advantages in correct covers detection in the complex street scene.
\end{abstract}

\section{INTRODUCTION}

Manhole cover is a necessary facility in urban design and traffic management. The missing of manhole may cause great security risks and economic losses, so the near real-time report of cover missing seems very important. There are many ways to prevent loses of covers. One way is to ensure the cover and road that are built as a whole entity with physical methods. Another way is to install a sensor with a transmitter in covers, which can report cover's position in real-time (Tang et al, 2003). However, the first way may cause some difficulties to get down to the underground for pipeline repair or draining. The second way may cause some additional needs of operations and economy. Cover detection and management with close-range images is a more popular way due to the development of computer vision science and photogrammetry.

Manhole covers have a more uniform appearance than other traffic features, such as traffic signs, traffic lights and zebra crossing It hasa relatively standard shape of circle or rectangle. The basic idea of cover detection is to find ellipses or rectangles in images and then determine if they are covers. Chen presents an ellipse detection method based on improved Hough transform (Chen and Wang, 2006). Liu utilises least squares fitting to find ellipses, and then the false targets are eliminated by parameters such as max and minor axis ratio and rotation angles (Liu, 2010).

However, the appearance of covers in images is not always a single and complete ellipse due to the complex street background, such as shadows of trees or buildings, the occlusion from cars or road railings. So in many cases only an arc of part ellipse can be found. In addition, some ellipse targets may not be covers such as wheels, traffic signs, etc.

In this paper we present an arc detection and multi-view matching method for cover detection, location and false target eliminating using close-range images, GPS/IMU and LIDAR data. In 2.1 the system workflow is briefly introduced and in 2.2 and 2.3 the arc detection, ellipse fitting and filter checking are presented. In 2.4 the multi-view matching is done to further check and locate the $3 \mathrm{D}$ position of manhole covers. In para. 3 we use 1100 images from vehicle-based camera with GPS/IMU, and LIDAR data to test our system and the results are discussed and in 4 the conclusion are drown.

\section{METHODOLOGY}

\subsection{Data and system workflow}

The vehicle based close-range imaging system Ladybug (Ladybug, 2012) is used as data acquisition equipment. There are six separate fisheye cameras in the car. No.0 4 cameras are around the car with 72 degree between each other and No.5 camera points to the sky and all camera can form a panoramic image in geometry (see Fig. 1). We use three front cameras No.0, 1 and 4 to detect manholes. GPS/IMU system with high accuracy is installed in the car to supply external orientation parameters (EOs) at the exposure time. The LIDAR data is also provided to get the road height.

The workflow in Fig. 2 includes two main steps, edge detection and texture detection. In image pre-process, the fisheye images

\footnotetext{
${ }^{*}$ Corresponding author.
} 
are resampled to ideal plane images using the camera calibration parameter supported by PGR Ladybug systems, and the interesting area is extracted for efficiency. The canny based on EDISON (Meer and Georgescu, 2001) edge detection method is used to detect arcs in the interesting area. Then each arc is fitted to the most possible ellipse using least square method. The direction, area and height information of extracted ellipse are then checked to exclude false detection such as shadows, wheels and traffic signs. In the texture verification step, the texture complexity is firstly estimated by variance of intensity in ellipse to exclude the area lack of texture. Then the multiview images are matched to further validation and at the same time the location of covers are obtained with GPS/IMU.

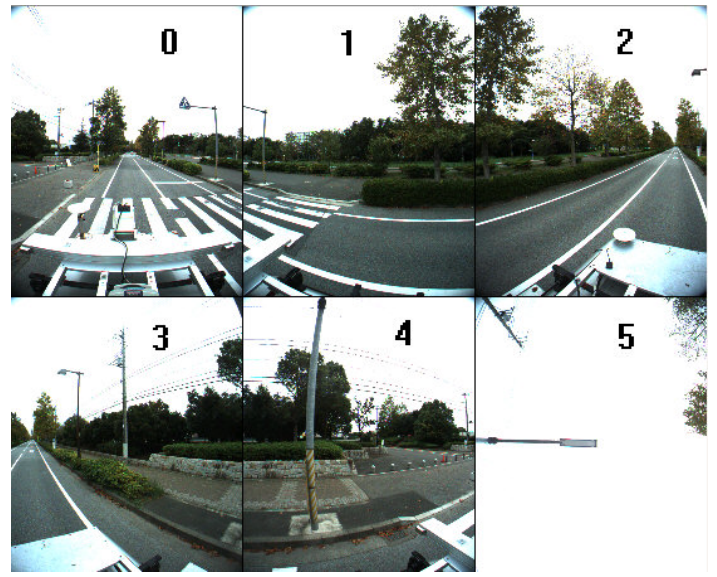

Figure 1. Separate fisheye images

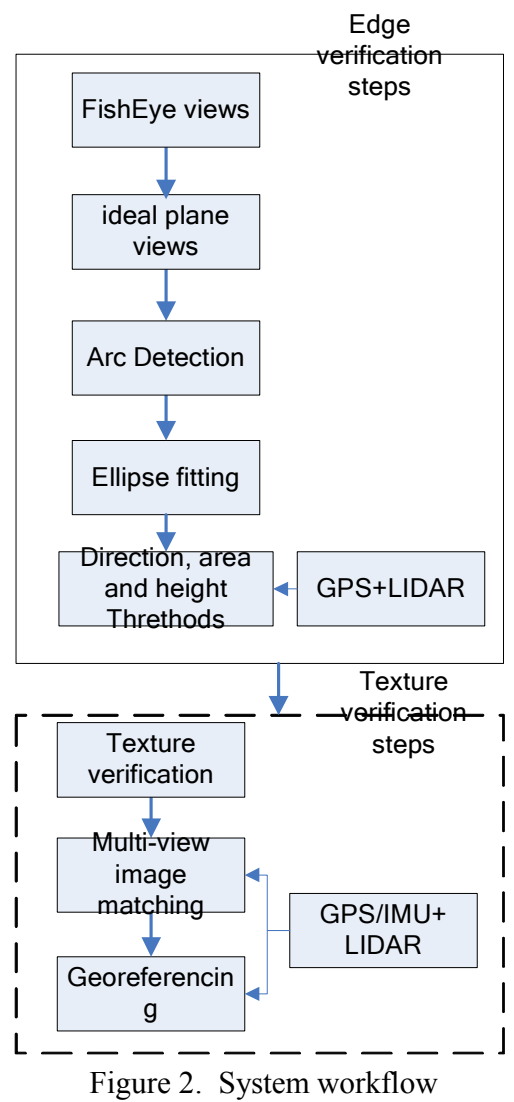

\subsection{Arc detection}

The shape of manhole is mainly ellipse or rectangle. However, those two shapes are symmetric, and same extraction method could be adapted to those two features. This paper mainly deals with manholes with ellipse shapes. For the reasons such as occlusion, shadows, illumination, blurring, the ellipse cover does not show whole and perfect ellipse, and in many cases only one part of ellipse can be discovered. Our method is to detect arcs firstly, and then finds the most possible ellipse after multi-step checking.

For efficiency, the area of $1024 * 527$ in pixels is extracted for the next processing. We use EDISON method to detect arcs and lines. The gradient window radius is set to 3 and the minimum length of arc is set to 40. The type of non-maximum suppression is set as vertical line and the type of hysteresis High threshold is set as horizontal line and low threshold as arc. All the values of rank and confidence take the default.

\subsection{Ellipse fitting and thresholds}

Most of the arcs been extracted do not belong to covers for the complex street scenes. The ellipse fitting is then used to fine the possible ellipses that agree to the proper shape and size of covers. The elliptic equation with unknown parameters as long axle $a$, minor axle $b$ and rotation angle was solved with RANSAC (Fischler and Bolles, 1981) method according to the points in the arcs.

After the ellipses are obtained some thresholds are then used to eliminate those ones which not fitting a cover. Because only the covers in front of the car are considered, long axle is likely rectangular to the road direction. So is limited to 45 degree. For the area restriction, long axle $a$ is set to between $0.5 \mathrm{~m}$ and $1 \mathrm{~m}$ and $b$ is set to $0.3 \mathrm{~m}$ and $0.9 \mathrm{~m}$ and $a>b$.

There are still some objects with elliptic shape in the interesting area, such as wheels and some traffic signs. The LIDAR observation values are used to eliminate those false arcs by height information. If the height of ellipse center is $0.2 \mathrm{~m}$ higher than the ground height, the ellipse will be excluded as false detection.

\subsection{Multi-view matching and locating}

Except shape, texture is another important feature for cover detection and false checking. In the paper matching method is utilised to confirm the candidate ellipses. Due to the deferent sight angle, deferent material and shadow, lamination etc, it is difficult to find a universal template to match all candidates correctly. In this paper, the multi-view matching for adjacent images are used instead, so as to confirm the detection results and to obtain the accrue geo-position of covers combined with GPS/IMU data.

Before matching the ellipse is verified to have enough texture information. The variance in pixel intensity within the ellipse should be more than 50 . However, there are some covers have less variance in intensity for the light situation (see Fig. 3d). In such situation the cover will be missed.

The covers have been taken at deferent distance to the car and there is identified deformation between those covers. The deformation formula can be described like eq. 2 deducted from eq. 1 when the cover is regarded as in a plane. In eq. $1, x_{1}$ and $X$ are the coordinate vector of reference image and ground coordinate respectively, and $R_{1}$ and $T_{1}$ are the rotation matrix and translation vector, which provided by GPS/IMU observations. $R_{2}, T_{2}$ and $x_{2}$ are the correspondent observations of an arbitrary matching image. Combined with LIDAR data, $\lambda$ can be calculated from the first formula in eq. 1 while $Z$ coordinate in $X$ is known. 


$$
\begin{gathered}
X=\lambda R_{1} x_{1}+T_{1} \\
x_{2}=R_{2}^{T}\left(X-T_{2}\right) \\
X=R_{1} x_{1}+T_{1} \\
x_{2}=R_{2}^{T}\left(\lambda R_{1} x_{1}+T_{1}-T_{2}\right)
\end{gathered}
$$

According to eq.2, all the matching images can be resampled to the reference image. Then the least square image matching is done between the patch of candidate covers in reference image and corresponding area in matching images.

Taking into account that there are some occlusions in covers and only a part of cover can be detected, the matching method deals with two situations. Firstly, the whole ellipse is matched and if the matching successfully, this step ends and if not, the part of ellipse according to the arc been detected by EDISON is then matched.

After matching, the floating objects such as wheels and floating shadows with round shape will be excluded.

Then the forward intersection of corresponding image points is done to get ground location with EOs supplied by GPS/IMU.
Due to the high accuracy of EOs, the matching result will be regard as failure if the REMS of intersection is more than $0.2 \mathrm{~m}$. In most cases, we consider two images for one single cover. The front cameras take two images with nearest cover presentation of $2 \mathrm{~m}$ interval in object space. The farer images are not used for relatively small and vague texture (see Fig. 5d). If the two images are correctly matched, we consider that's a successful cover detection and location.

\section{EXPERIMENTS}

\subsection{Test data}

The test data include 1100 images and 26 distinct manhole covers. In this test the manhole covers exhibit different textures, sizes, and different view points, especially the influence by shadows and occlusions. The positioning accuracy of GPS data is about $0.05 \mathrm{~m}$ and the rotate angle accuracy of IMU is more than 30 . The locating accuracy of LIDAR is more than $0.05 \mathrm{~m}$ within $60 \mathrm{~m}$
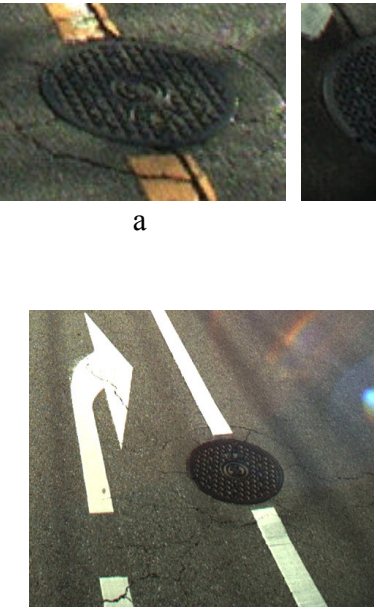

a

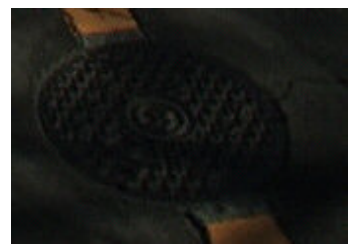

a

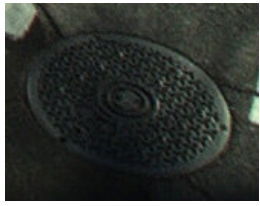

b

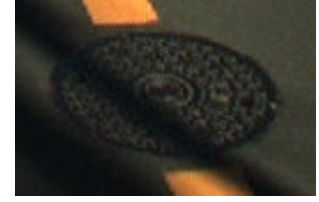

$\mathrm{c}$

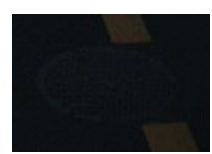

d

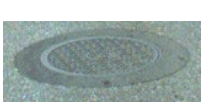

e

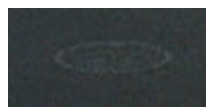

f

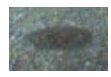

g

Figure 3. Variety of manhole covers

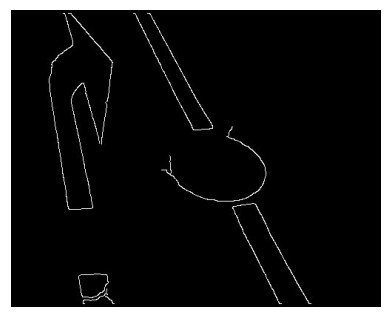

b

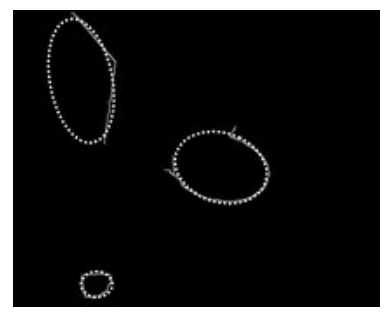

c

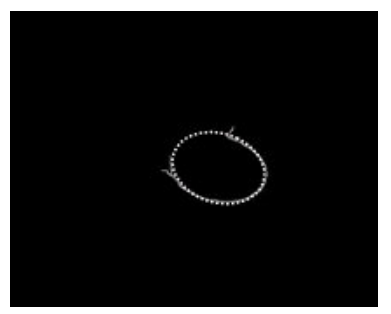

d

Figure 4. Edge detection

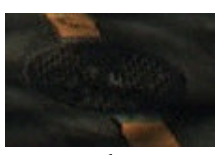

b

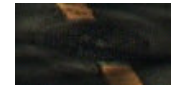

$\mathrm{c}$

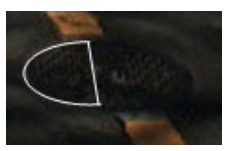

d

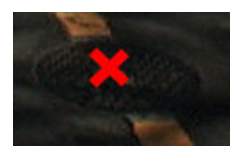

Figure 5. Matching and locating

\subsection{Edge detection evaluation}

After the interesting area is extracted the EDISON edge extraction is done to find edges which may contain covers, see figure $4 \mathrm{a}, 4 \mathrm{~b}$. Then the ellipses are fitted for each independent edge. If more than $60 \%$ points in edge segment is fitted to an ellipse with REMS less than 2 pixels, we consider it a successful fitting. In fig. $4 c$, there are 3 edges can fitted to an ellipse among 7 segments. Obviously, there are two fitted ellipses are not belong to covers but belong to traffic lines. In the next step, the area and direction constraints are used to exclude those two ellipses, and in fig. $4 \mathrm{~d}$ only the right ellipse corresponding to the cover remains.

The red line in fig. 6a shows the missing rate via false positives (FP) rate only use edge information. With the FP increases the missing rate decreases but slowly. In the FP rate of 0.05 the detection rate is $79 \%$. It should be noted that the missing covers which cannot be extracted by EDISON also been included in the statistics.

\subsection{Matching and location evaluation}

The adjacent images with candidates are then matched for further confirm and for locating. The part and whole ellipse are separately matched according to the arcs been extracted. Firstly the whole ellipse is matched only in two adjacent images for the third images about $6 \mathrm{~m}$ away lose most of the texture (see Fig. $5 \mathrm{c}$ ). If the matching success, part arcs will not be matched. The fig. $5 \mathrm{~d}$ shows the situation that the whole ellipse can not match but the part in the arc (white line) can match. The Fig. 5e shows 
that the successfully matched covers are confirmed and the location is calculated from forward intersection with GPS/IMU data. However, the cover location is taken from the center of ellipse, it may be a slightly deviation from the actual center of the cover.

The blue line in fig. 6a shows the missing rate via false positives (FP) rate use edge and texture information. The detection rate has a little increase as $88 \%$ in 0.05 FP rate for some round shape objects lack of texture are eliminated. More ever, the covers are located precisely due to the high accuracy of GPS and IMU data after matching.

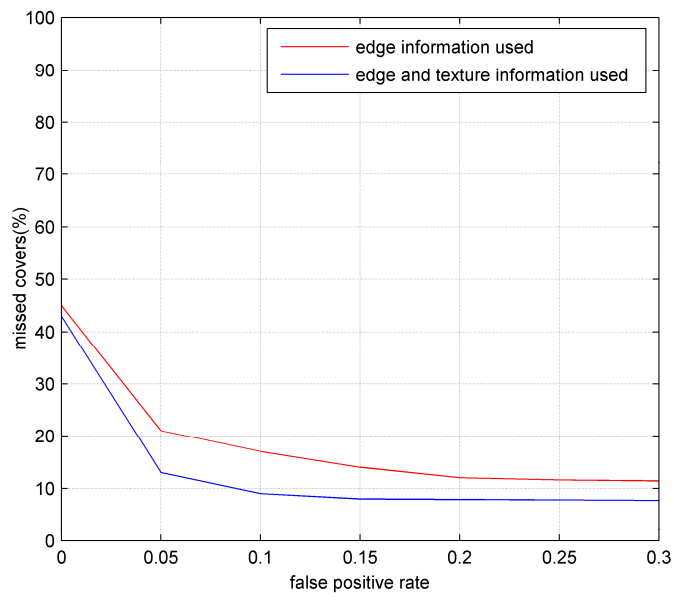

Figure 6 . The detection performance based only on edge information and on edge and texture

\subsection{Discussion}

This work investigated both detection and location of the manhole covers using edge and texture information. The test shows a good performance in covers detection in different situation under muti-view, occlusions, shadows, sizes.

The simple ellipse fitting method (Chen and Wang, 2006; Liu, 2010) cannot deal with occlusions and false round objects. More information and process steps should be involved to decrease the FP.

The single-view and multi-view combined method (Radu and Luc, 2011) has a high detection rate for more reliable information such as edges, area, symmetry, been added to detect and locate covers. However, the detection rate does not consider the missed covers which cannot be properly segmented by mean-sift segmentation method.

As mentioned in the above paper (Radu and Luc, 2011), the RFID tagging base method (Chang et al, 2009) need additional operation for tagging all the covers and it is not easy to a fast and convenient browse of all the covers.

Of course, our method also has problems. The biggest problem is that the following steps all depend on the edge detection method. In the special circumstances as fig. $3 \mathrm{~d}$, it is difficult for edge detection methods to find proper edges since the intensity from material of the cover and of the road are very similar. For the further increasing of detection rate the very weak edge detection should be solved firstly.

\section{CONCLUSIONS}

Manhole cover detection is a more challenging problem than expected that many disadvantageous situations should be considered. In this paper the vehicle-based cameras combined with GPS, IMU, LIDAR is used to detect and locate the manholes in streets. The canny based EDISON edge detection method and texture based matching method are both used in our method to assure a high detection rate. The matching result is also used to accurately calculate the $3 \mathrm{D}$ position of covers combined with GPS/IMU data.

The future work will focus on the weak edge detection and some other close-range applications using our method, such as traffic signs detection, traffic lights detection, etc.

Acknowledgements. This work has been partly supported by Chinese 973 program (2012CB719902).

\section{References}

Chang, A.Y., Yu, C.S., Lin, S.C., Chang, Y.Y. and Ho, P.C., 2009. Search, identification and positioning of the underground manhole with rfid ground tag. Networked Computing and Advanced Information Management, International Conference on, pp. 1899-1903.

Chen, Y., Xiong, Z. and Wang, Y.H., 2006. Improved classical hough transform applied to the manhole cover's detection and location. Optical Technique (in Chinese), 32(2006), pp. 504508.

Fischler, M.A. and Bolles, R. C., 1981. Random Sample Consensus: A Paradigm for Model Fitting with Applications to Image Analysis and Automated Cartography. $C A C M, 24(6)$, pp. 381-395.

Liu, J.H., 2011. Research on algorithm of automatically recognizing and positioning road manhole covers based on vehicle- mounted sensors. Application Research of Computers (in Chinese), 28(8), pp. 3138-3140.

Mer, P. and Georgescu, B., 2001. Edge detection with embedded confidence. IEEE Transactions on Pattern Analysis and Machine Intelligence, 23(12), pp. 1351-1365.

Radu, T. and Luc, V.G., 2011. Multi-view manhole detection, recognition, and 3D localisation. IEEE International Conference on Computer Vision Workshops 2011, pp. 188-195. Tang, N, 2003. Manhole detection and location for urban pavement. IEEE transportation system, 2(12), 1552-1555.

LADYBUG, http://www.ptgrey.com/products/spherical.asp. (2 Mar. 2012) 